\title{
Comportamento da interação genótipos por locais aos três e nove anos em clones de eucalipto
}

\author{
Genotype by environmental interaction pattern over three and nine \\ years in Eucalyptus clones
}

\author{
Carla Aparecida de Oliveira Castro', Andrei Caíque Pires Nunes², \\ Osmarino Pires dos Santos ${ }^{3}$, Rafael Tassinari Resende ${ }^{4}$, \\ Gleison Augusto dos Santos ${ }^{5}$, Marcos Deon Vilela de Resende ${ }^{6}$ e Cosme Damião Cruz ${ }^{1}$
}

\begin{abstract}
RESUMO
O presente trabalho teve como objetivo determinar o padrão de estratificação ambiental em um estudo de interação $G \times A$ de plantios clonais de eucalipto, aos 3 e 9 anos. Os experimentos foram conduzidos nos municípios Encruzilhada do Sul (horto Capivara), Dom Feliciano (horto Fortaleza) e Vila Nova do Sul (horto São João), no Rio Grande do Sul. Na totalidade, foram utilizados 804 clones de eucalipto nos testes, plantados em espaçamento de 3,5 $\mathrm{m} \times 2,6 \mathrm{~m}$. Foi empregado o delineamento de blocos ao acaso, com parcela de árvore única e 30 repetições. Como testemunha, foi utilizado o clone comercial 32864 (Eucalyptus saligna). $O$ diâmetro à altura do peito, a altura total das árvores e a sobrevivência, foram mensurados aos 3 e 9 anos. $\mathrm{O}$ volume e o incremento médio anual (IMA) dos testes foram calculados a partir destes dados coletados. A partir dos dados citados, referentes às duas idades, foram realizadas análises genéticas via metodologia de modelos mistos, utilizando o software Selegen REML/BLUP. Constatou-se que o padrão de interação $G \times A$ modificou dos 3 para os 9 anos, devido à uniformização do desempenho dos clones e à melhor adaptação destes às variações ambientais em idades mais avançadas. A maior uniformidade foi expressa pelo aumento das correlações genotípicas entre os locais nas diferentes idades. Aos 3 anos foram encontradas três zonas de melhoramento, representadas por cada sítio, enquanto que aos 9 formaram-se duas zonas, sendo estas compostas pelos hortos São João-Fortaleza e Capivara. A partir do ordenamento dos materiais genéticos superiores nos ambientes, para o caráter IMA, por meio da análise conjunta nas duas idades, concluiu-se que os cinco melhores clones apresentaram desempenho superior em relação à testemunha, com ganho acumulado de $21 \%$, e em relação à média da população.
\end{abstract}

Palavras-Chave: Melhoramento florestal; Interação Genótipos × Ambientes $(G \times A)$; Testes clonais; Recomendação genotípica.

\section{ABSTRACT}

This study aimed to determine the environmental stratification pattern in a $G \times E$ interaction study of three clonal Eucalyptus trials at 3 and 9 years after planting. The experiments were conducted in the cities of Encruzilhada do Sul (Capivara site), Dom Feliciano (Fortaleza site) and Vila Nova do Sul (São João site), in Rio Grande do Sul. In total, 804 Eucalyptus clones were used in the tests, planted at spacing of $3.5 \mathrm{~m} \times 2.6 \mathrm{~m}$. A complete randomized block design with single tree plot and 30 replicates was used. As a control, commercial clone 32864 (Eucalyptus saligna) was used. The diameter at breast height, total tree height and survival were measured at 3 and 9 years. Volume and mean annual increment (MAI) of the tests were calculated from these data. From the cited data, genetic analyses were carried out by mixed models methodology, using Selegen REML / BLUP software. It was verified that the interaction pattern $G \times E$ changed from 3 to 9 years, due to the uniformity of clones performance and the better adaptation of these materials to the environmental variations at more advanced ages. The greater uniformity was expressed by the increase in genotype correlations between sites at different ages. At the age of 3 years three breeding areas were found, represented by each site, while in the 9 age there were two zones, these being composed

\footnotetext{
1.Departamento de Ciências Florestais, Universidade Federal de Viçosa - UFV. Viçosa / MG, Brasil. E-mail: carla.castro0120@gmail.com.com

2. Universidade Federal do Sul da Bahia - UFSB. Itabuna / BA, Brasil.

3. CMPC Celulose Riograndense. Gauíba / RS, Brasil.

4. Escola de Agronomia / Engenharia Florestal, Universidade Federal de Goiás - UFG. Goiânia / GO, Brasil.

5. Departamento de Engenharia Florestal, Universidade Federal de Viçosa - UFV. Viçosa / MG, Brasil.

6. Centro Nacional de Pesquisa de Florestas, Empresa Brasileira de Pesquisa Agropecuária - Embrapa. Colombo / PR, Brasil.
}

Sci. For., Piracicaba, v. 46, n. 120, p. 594-605, dez. 2018 DOI: dx.doi.org/10.18671/scifor.v46n120.08 
by the sites of São João-Fortaleza and Capivara. From the ordering of the superior genetic materials in the environments, for the IMA character, through the joint analysis in the two ages, it was concluded that the five best clones presented superior performance in relation to the control, with an accumulated gain of $21 \%$, and above the population mean.

Keywords: Forest breeding; Genotype by Environment Interaction $(\mathrm{G} \times \mathrm{E})$; Clonal trials; Genetic Selection.

\section{INTRODUÇÃO}

A existência de grandes maciços florestais, constituídos de espécies do gênero Eucalyptus, garante a matéria-prima necessária para a produção de diferentes produtos madeireiros presentes no cotidiano da população. Os avanços tecnológicos envolvendo a ciência florestal são responsáveis pelo estabelecimento de plantios clonais de eucalipto de alta produtividade e que demandam menor tempo de rotação e de custo para a produção de madeira, quando comparado às outras espécies madeireiras (BIRK; TURNER, 1992; FONSECA et al., 2010). Melhorias contínuas na qualidade dos produtos resultantes de plantios de eucalipto têm sido obtidas, em virtude dos investimentos em programas de melhoramento genético, feitos por empresas e instituições de ensino e pesquisa nos últimos anos (ARAÚJO et al., 2015; ASSIS; ABAD; AGUIAR, 1996; CASTRO et al., 2016).

Os programas de melhoramento genético florestal baseiam-se na seleção e recombinação de materiais genéticos superiores, visando a melhoria das condições adaptativas e o aumento da frequência dos alelos de interesse (PIRES et al., 2011). Para determinar quais são os clones mais adaptados aos diferentes ambientes, são necessárias avaliações de produtividade em regiões distintas de plantio da rede experimental (CRUZ; REGAZZI; CARNEIRO, 2012). A partir desses estudos, é possível compreender o desempenho ao longo do tempo nestas regiões (ODA; MENCK; VENCOVSKY,1989).

Assim como avaliar o desempenho dos genótipos ao longo do tempo, é relevante considerar as condições ambientais em que estes estão estabelecidos (CRUZ; REGAZZI; CARNEIRO, 2012; HARDNER et al., 2011; VENCOVSKY; BARRIGA, 1992). A interação genótipos por ambientes ( $\mathrm{G} \times \mathrm{A}$ ) pode provocar alterações na magnitude e significância das variâncias que compõem os parâmetros genéticos da população, assim como mudanças no ordenamento dos melhores indivíduos no gradiente ambiental (FALCONER et al., 1996).

A interação $G \times$ A pode ser classificada de duas maneiras, sendo estas denominadas como simples e complexa. A interação simples é caracterizada pela ausência de alterações das posições relativas dos genótipos, dentro de um conjunto de ambientes, avaliados dois a dois. A complexa, ocorre quando a correlação entre o desempenho dos genótipos ao longo dos ambientes em estudo é baixa, alterando a posição relativa dos genótipos em virtude das diferentes respostas às variações ambientais (CRUZ; REGAZZI; CARNEIRO, 2012; GARBUGLIO et al., 2007). A existência de interação G × A na rede experimental, pode indicar que os melhores clones em um ambiente não apresentarão bom desenvolvimento em outro (MALOSETTI et al., 2013; NUNES et al., 2002; PIRES et al., 2011). Desta maneira, avaliar a existência de interação $\mathrm{G} \times \mathrm{A}$ é fundamental para a maximização de ganhos genéticos ao final do programa de melhoramento florestal, sendo portando, uma etapa imprescindível a ser realizada pelo melhorista (HARDNER et al., 2011). Além disso, é necessário o estudo do padrão de interação $G \times A$ em avaliações periódicas, uma vez que esta interação é determinante para a manifestação do fenótipo e pode se alterar com o passar dos anos (NUNES et al., 2002; CRUZ; REGAZZI; CARNEIRO, 2012).

Outro aspecto importante no direcionamento dos programas de melhoramento, é a obtenção de estimativas dos parâmetros genéticos, pois estas auxiliam no processo de seleção e na recomendação de novos clones comerciais (MAIA et al., 2009). Devido a esta relevância, é fundamental a análise adequada de parâmetros, para compreender a magnitude e natureza da interação entre clones e ambientes, permitindo então, a escolha da melhor estratégia de seleção (NUNES et al., 2016; 2002).

O objetivo deste trabalho foi determinar o padrão de estratificação ambiental em um estudo de interação $\mathrm{G} \times \mathrm{A}$ em plantios clonais de eucalipto, presentes em três localidades, no estado do Rio Grande do Sul. 
Castro et al. - Se Comportamento da interação genótipos por locais aos três e

nove anos em clones de eucalipto

\section{MATERIAL E MÉTODOS}

\section{Áreas experimentais}

Os experimentos foram conduzidos nas áreas da empresa CMPC Celulose Riograndense, nos municípios de Encruzilhada do Sul (Horto Florestal Capivara, lat $30^{\circ} 27^{\prime} \mathrm{S}$, long $52^{\circ} 39^{\prime} \mathrm{W}$ ), Dom Feliciano (Horto Florestal Fortaleza, 30 $29^{\prime}$ S, long 52 ${ }^{\circ} 19^{\prime} \mathrm{W}$ ) e Vila Nova do Sul (Horto Florestal São João, lat $30^{\circ} 14^{\prime}$ S, long $53^{\circ} 49^{\prime}$ W), todos localizados no estado do Rio Grande do Sul, Brasil (Figura 1).
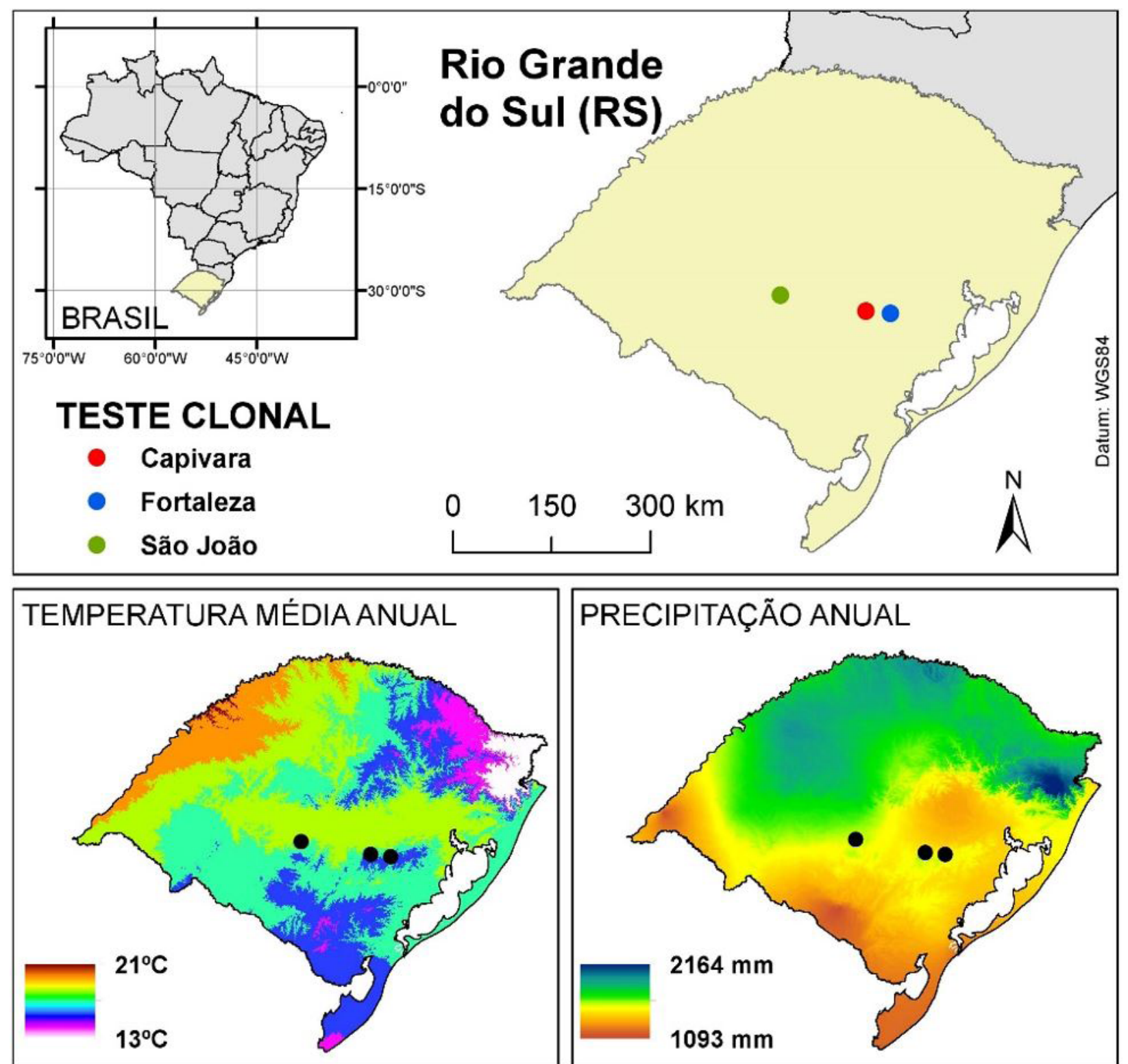

Figura 1. Ambientes que compõem a rede experimental (Capivara, Fortaleza e São João). Os mapas inferiores representam as médias de temperatura e precipitação anual do estado do Rio Grande do Sul, com destaque para os locais de interesse.

Figure 1. Environments that compose the experimental network (Capivara, Fortaleza and São João). The lower maps represent the averages of temperature and annual precipitation of the state of Rio Grande do Sul, with emphasis on the sites of interest.

Além das condições climáticas, outro fator relevante levantado em relação às áreas em estudo, foi a classificação do solo em cada sítio (Tabela 1). 
Tabela 1. Condições edafoclimáticas de três ambientes do estado do Rio Grande do Sul, utilizados para experimentação.

Table 1. Edaphoclimatic conditions of three environments on the state of Rio Grande do Sul, Brazil, used for trial.

\begin{tabular}{|c|c|c|c|}
\hline \multirow{2}{*}{ Município } & Capivara & Fortaleza & São João \\
\hline & Encruzilhada do Sul & Dom Feliciano & Vila Nova do Sul \\
\hline Tipo de Solo & $\begin{array}{c}\text { Argissolo Vermelho } \\
\text { distrófico } \\
\text { abrúptico - } \\
\text { Textura arenosa/argilosa }\end{array}$ & $\begin{array}{c}\text { Argissolo Vermelho } \\
\text { Amarelo } \\
\text { distrófico típico - } \\
\text { Textura média/argilosa }\end{array}$ & $\begin{array}{c}\text { Argissolo Vermelho } \\
\text { distrófico } \\
\text { típico - } \\
\text { Textura média/argilosa }\end{array}$ \\
\hline Fertilidade & Média a alta & Média a alta & Média a baixa \\
\hline Profundidade do solo & Médio & Raso & Profundo \\
\hline Relevo & Ondulado & Ondulado & Plano suave ondulado \\
\hline $\begin{array}{l}\text { Mudança textural } \\
\text { (perfil do solo) }\end{array}$ & Moderada & Forte & Leve \\
\hline Temperatura Média & $17,0^{\circ} \mathrm{C}$ & $16,0^{\circ} \mathrm{C}$ & $16,8^{\circ} \mathrm{C}$ \\
\hline Pluviosidade & $1.368 \mathrm{~mm}$ & $1.564 \mathrm{~mm}$ & $1.133 \mathrm{~mm}$ \\
\hline
\end{tabular}

Adaptado de Santos et al. (2013).

\section{Delineamento experimental e coleta de dados}

A rede de testes clonais foi implantada no ano de 2007 com 804 clones de eucalipto (a descrição detalhada das espécies encontra-se no artigo de SANTOS et al., 2016), plantados em espaçamento de $3,5 \mathrm{~m} \times 2,6 \mathrm{~m}$. Como testemunha foi utilizado o principal clone comercial plantado pela empresa (clone 32864, E. saligna). Em cada sítio, um experimento com delineamento de blocos ao acaso (DBC) foi estabelecido, com parcelas de árvore única e 30 repetições.

O número total de clones em cada ambiente variou, sendo que, em Capivara foram plantados 515 clones, em Fortaleza haviam 561 e em São João, 579. A quantidade de clones em comum entre Capivara e Fortaleza foi de 345, Capivara e São João, 365 e Fortaleza e São João, 413. Os 115 clones comuns em todos os ambientes foram utilizados para a análise conjunta da interação $\mathrm{G} \times \mathrm{A}$.

A coleta dos dados foi realizada aos 3 e 9 anos de idade, mensurando-se o diâmetro à altura do peito (DAP), em centímetros, e a altura total das árvores (Ht), em metros. O DAP foi mensurado com o auxílio de uma fita diamétrica e a altura foi obtida com o uso do relascópio. Além disso, foi analisada e quantificada a sobrevivência nos testes.

O volume total da árvore $\left(\mathrm{Vol}, \mathrm{m}^{3}\right)$ foi calculado nos dois períodos, seguindo a equação de Schumacher e Hall (1933). O cálculo do incremento médio anual (IMA, em m . ha $^{-1}$. $\mathrm{ano}^{-1}$ ), foi efetuado utilizando o volume individual das árvores extrapolado para 1 ha e dividido pela idade.

\subsection{Modelos estatísticos}

A partir dos dados coletados, foram realizadas diferentes análises utilizando modelos do software Selegen REML/BLUP (RESENDE, 2016). Estes modelos foram usados com o objetivo de avaliar o comportamento das populações clonais nos dois períodos, o padrão de interação $\mathrm{G} \times \mathrm{A}$ com o passar dos anos e a ocorrência de diferenças na expressão dos valores genotípicos dos clones entre os ambientes.

\subsubsection{Modelo estatístico para avaliação da interação G x A em cada idade}

A interação $\mathrm{G} \times \mathrm{A}$ foi avaliada segundo o modelo abaixo:

$$
Y=X r+Z g+W b+T i+e
$$

Em que Y é o vetor de dados; r é o vetor dos efeitos fixos (média geral neste caso), ajustando combinações média-local; $\mathbf{g}$ é o vetor dos efeitos genotípicos (assumidos como aleatórios); b é o vetor dos efeitos de blocos (assumidos como aleatórios); i é vetor dos efeitos da interação genótipo $\mathrm{x}$ ambiente (aleatórios); e é o vetor de erros ou resíduos (aleatórios). As letras maiúsculas ( $\mathbf{X}, \mathbf{Z}, \mathbf{W}$ e T) representam as matrizes de incidência para os referidos efeitos.

\subsubsection{Modelo estatístico para análise de teste clonal por local em cada idade}

A análise local em cada ano foi realizada, conforme exposto abaixo:

$$
Y=X r+Z g+W b+e
$$


Em que Y é o vetor de dados; r é o vetor dos efeitos fixos (média geral neste caso); $\mathbf{g}$ é o vetor dos efeitos genotípicos (assumidos como aleatórios); b é o vetor dos efeitos de blocos (assumidos como aleatórios); e é o vetor de erros ou resíduos (aleatórios). As letras maiúsculas ( $\mathbf{X}, \mathbf{Z} \in \mathbf{W}$ ) representam as matrizes de incidência para os referidos efeitos.

\subsubsection{Modelo estatístico para avaliar a rede experimental nos três ambientes e nas duas idades}

A avaliação de toda a rede experimental, considerando as informações de todas as medições, ocorreu de acordo com o modelo abaixo:

$$
Y=X f+Z g+O g l+T g m+W g l m+S p+e
$$

Em que Y é o vetor de dados; fé o vetor dos efeitos das combinações média-local-medição (assumidos como fixos); g é o vetor dos efeitos genotípicos (assumidos como aleatórios); gl é vetor dos efeitos da interação de genótipos com locais (aleatórios); gm é o vetor dos efeitos da interação genótipos $\mathrm{x}$ medições; glm é o vetor dos efeitos da interação tripla genótipos x locais x medições (assumidos como aleatórios); $\mathbf{p}$ é o vetor dos efeitos permanentes de parcela dentro de locais (assumidos como aleatórios); e é o vetor de erros ou resíduos (aleatórios). As letras maiúsculas ( $\mathbf{X}, \mathbf{Z}, \mathbf{O}, \mathbf{T}, \mathbf{W}$ e $\mathbf{S}$ ) representam as matrizes de incidência para os referidos efeitos.

\subsection{Análise de ganhos genéticos, estabilidade e adaptabilidade}

A partir dos resultados obtidos por meio do modelo estatístico que permitiu a análise simultânea da rede experimental entre todos os locais e idades, foram encontrados os valores genotípicos dos clones. Desta forma, foi realizado o ranqueamento dos clones superiores na amostragem em relação aos ganhos genéticos obtidos em relação à média geral da população e à testemunha (clone 32864). Este comparativo baseou-se na análise de produtividade dos clones (IMA), avaliando os valores genotípicos preditos livres de interação $(\mathrm{u}+\mathrm{g})$ e os valores genotípicos preditos acrescidos da interação média entre ambientes ( $\mathrm{u}+\mathrm{g}+\mathrm{gem})$.

Além disso, com os valores genotípicos preditos para o caráter IMA, os genótipos foram ranqueados de acordo com a seleção conjunta por produtividade, estabilidade e adaptabilidade. Este procedimento foi baseado na estatística denominada média harmônica da performance relativa dos valores genéticos (MHPRVG*MG) preditos, conforme descrito por Resende (2004). As análises citadas também foram realizadas utilizando o software Selegen REML/BLUP.

\section{RESULTADOS E DISCUSSÃO}

\section{Parâmetros genéticos da análise de interação $\mathbf{G} \times \mathbf{A}$ por idade}

As estimativas de herdabilidade individual no sentido amplo para os caracteres, aumentaram de 3 para 9 anos (Tabela 2). Estes acréscimos variaram entre 39\% (DAP) a 161\% (Ht). De acordo com Resende (2002), pode-se considerar que a herdabilidade variou entre valores de baixa $\left(\mathrm{h}^{2} \mathrm{~g}\right.$ entre $0,01$ e 0,15$)$ a média $\left(\mathrm{h}^{2} \mathrm{~g}\right.$ entre 0,15 e 0,50$)$ magnitude nos dois períodos, para todo os caracteres. A sobrevivência apresentou herdabilidade baixa nas duas idades $\left(\mathrm{h}^{2} \mathrm{~g}=0,02\right.$ e 0,03$)$, o que indica que este é um caráter fortemente influenciado pelo ambiente.

A herdabilidade de médias de clones $\left(\mathrm{h}^{2} \mathrm{mg}\right)$ para o IMA, avaliado de forma conjunta nos três ambientes, apresentou valores de média a alta magnitude, que foram iguais a 0,58 e 0,74, quando obtidos aos 3 e 9 anos, respectivamente. Estes valores demonstram que há potencial em se realizar a seleção a partir de médias de clones. A $\mathrm{h}^{2} \mathrm{mg}$ é estimada quando se usam médias como unidade de avaliação e de seleção e possui maior nível de precisão, em razão da diminuição dos erros experimentais e incremento do número de repetições (ROSADO et al., 2012).

$\mathrm{O}$ coeficiente de variação relativa $(\mathrm{CVr})$ apresentou valores baixos a moderados, variando entre 0,14 (Sobrevivência) a 0,64 (DAP). O CVr para todos caracteres aumentou dos 3 aos 9 anos, sugerindo que as populações apresentaram maior variabilidade genética aos 9 anos.

As acurácias dos valores genéticos preditos variaram de 0,62 (Ht) a 0,87 (DAP), sendo estes valores considerados médios a altos, de acordo com Resende e Duarte (2007). Para o caráter IMA, as acurácias apresentaram valores elevados iguais a 0,76 e 0,86 aos 3 e 9 anos, respectivamente, corroborando com resultados obtidos por Resende et al. (2017). Esses autores demonstraram altas 
Tabela 2. Estimativas de parâmetros genéticos, para os caracteres diâmetro à altura do peito (DAP em cm), altura total ( $\mathrm{Ht} \mathrm{em} \mathrm{m}$ ), volume (Vol em $\left.\mathrm{m}^{3}\right)$, incremento volumétrico médio anual (IMA em $\mathrm{m}^{3}$.ha ${ }^{-1}$.ano ${ }^{-1}$ ) e sobrevivência de clones de eucalipto, avaliados na análise conjunta entre os ambientes aos 3 e 9 anos de idade.

Table 2. Estimates of genetic parameters, for traits diameter at breast height (DAP in $\mathrm{cm}$ ), total height (Ht in $\mathrm{m}$ ), volume $\left(\mathrm{Vol}\right.$ in $\mathrm{m}^{3}$ ), mean annual increment (MAI in $\mathrm{m}^{3}$. ha-1. $\mathrm{yr}^{-1}$ ) and individual survival of Eucalyptus clones, evaluated in the joint analysis between environments at 3 and 9 years.

\begin{tabular}{|c|c|c|c|c|c|c|c|c|c|c|}
\hline \multirow{2}{*}{ Parâmetros } & \multicolumn{2}{|c|}{ DAP } & \multicolumn{2}{|c|}{$\mathrm{Ht}$} & \multicolumn{2}{|c|}{ Vol } & \multicolumn{2}{|c|}{ IMA } & \multicolumn{2}{|c|}{ Sobrevivência } \\
\hline & 3 & 9 & 3 & 9 & 3 & 9 & 3 & 9 & 3 & 9 \\
\hline$h^{2} g$ & 0,18 & 0,24 & 0,06 & 0,16 & 0,14 & 0,23 & 0,14 & 0,23 & 0,02 & 0,03 \\
\hline$h^{2} m g$ & 0,67 & 0,76 & 0,41 & 0,73 & 0,58 & 0,74 & 0,58 & 0,74 & 0,38 & 0,47 \\
\hline Acgen & 0,82 & 0,87 & 0,64 & 0,85 & 0,76 & 0,86 & 0,76 & 0,86 & 0,62 & 0,68 \\
\hline $\mathrm{c}^{2}$ bloc & 0,04 & 0,01 & 0,08 & 0,01 & 0,05 & 0,01 & 0,05 & 0,01 & 0,03 & 0,02 \\
\hline$c^{2}$ int & 0,15 & 0,15 & 0,13 & 0,12 & 0,17 & 0,16 & 0,17 & 0,16 & 0,04 & 0,06 \\
\hline Rgloc & 0,53 & 0,62 & 0,32 & 0,58 & 0,45 & 0,59 & 0,45 & 0,59 & 0,30 & 0,34 \\
\hline CVgi\% & 8,20 & 15,00 & 5,02 & 10,91 & 17,97 & 34,70 & 17,97 & 34,70 & 5,22 & 7,64 \\
\hline CVe\% & 15,52 & 23,37 & 17,33 & 23,05 & 38,59 & 56,89 & 38,59 & 56,89 & 36,51 & 41,62 \\
\hline $\mathrm{CVr}$ & 0,53 & 0,64 & 0,29 & 0,47 & 0,47 & 0,61 & 0,47 & 0,61 & 0,14 & 0,18 \\
\hline Média & 13,17 & 19,51 & 14,43 & 26,48 & 0,10 & 0,44 & 42,77 & 65,14 & 0,86 & 0,83 \\
\hline
\end{tabular}

Sendo os parâmetros: $\mathbf{h}^{2} \mathbf{g}$ : herdabilidade de parcelas individuais no sentido amplo, ou seja, dos efeitos genotípicos totais; $\mathbf{h}^{2} \mathbf{m g}$ : herdabilidade ajustada da média de genótipo; Acgen: acurácia da seleção de genótipos; $\mathbf{c}^{2} \mathbf{b l o c}$ : coeficiente de determinação dos efeitos de bloco; $\mathbf{c}^{2} \mathbf{i n t}$ : coeficiente de determinação dos efeitos da interação genótipo x ambiente; Rgloc: correlação genotípica entre os ambientes; CVgi\%: coeficiente de variação genotípica individual; CVe\%: coeficiente de variação residual; CVr: coeficiente de variação relativa; Média geral do experimento.

Para todas as características o teste LRT apontou significância estatística para a herdabilidade (tabela de Deviance em materiais suplementares).

acurácias preditivas realizadas por meio de validação cruzada em testes clonais aos 6 anos para o mesmo caráter. Este resultado evidencia a alta confiabilidade dos valores genotípicos preditos e a qualidade experimental.

A correlação genética entre locais (Rgloc) apresentou valores abaixo de 0,70, demonstrando que os clones apresentaram desempenho diferenciado nos 3 ambientes. Neste caso, a recomendação generalizada de clones advindos de seleção precoce aos 3 anos, para os locais avaliados, não é indicada. Aos 9 anos, os valores de Rgloc aumentaram, demonstrando que as variações entre os ambientes diminuem com o tempo, pois são menos influenciados pelo ambiente aos 9 anos. Quanto maior for Rgloc, menor será a interação $G \times A$.

A diminuição dos valores do coeficiente de determinação dos efeitos da interação $\mathrm{G} \times \mathrm{A}\left(\mathrm{c}^{2}\right.$ int $)$ de 3 para 9 anos, corroboram com os resultados de Rgloc e com a teoria de que a interação $\mathrm{G} \times \mathrm{A}$ diminui com o tempo. $\mathrm{O} c^{2}$ int quantifica quanto da variação fenotípica é representada por efeito deste tipo de interação (STURION; RESENDE, 2005).

A partir da análise genética de cada local (Tabela 3) constatou-se que o sítio São João foi o mais produtivo dentre os avaliados (IMA igual a 50,27 e 74,37 $\mathrm{m}^{3} \cdot \mathrm{ha}^{-1}$.ano ${ }^{-1}$, aos 3 e 9 anos, respectivamente).

Tabela 3. Estimativas de parâmetros genéticos, relacionadas ao caráter incremento volumétrico médio anual (IMA em $\left.\mathrm{m}^{3} \cdot \mathrm{ha}^{-1} \cdot \mathrm{ano}^{-1}\right)$, para clones de eucalipto avaliados na análise de teste clonal individual em cada local aos 3 e 9 anos de idade.

Table 3. Estimates of genetic parameters, related to mean annual increment (MAI in $\mathrm{m}^{3} \cdot \mathrm{ha}^{-1} \cdot \mathrm{yr}^{-1}$ ) trait for Eucalyptus clones, evaluated in clonal trial analysis at each site in 3 and 9 years.

\begin{tabular}{|c|c|c|c|c|c|c|}
\hline \multirow{3}{*}{ Parâmetros } & \multicolumn{6}{|c|}{ IMA } \\
\hline & \multicolumn{2}{|c|}{ Capivara } & \multicolumn{2}{|c|}{ Fortaleza } & \multicolumn{2}{|c|}{ São João } \\
\hline & 3 & 9 & 3 & 9 & 3 & 9 \\
\hline$h^{2} g$ & 0,39 & 0,47 & 0,27 & 0,35 & 0,26 & 0,31 \\
\hline $\mathrm{c}^{2}$ bloc & 0,05 & 0,01 & 0,11 & 0,02 & 0,04 & 0,01 \\
\hline$h^{2} \mathrm{mc}$ & 0,41 & 0,48 & 0,30 & 0,35 & 0,27 & 0,32 \\
\hline Acclon & 0,97 & 0,98 & 0,96 & 0,97 & 0,94 & 0,94 \\
\hline CVgi\% & 27,29 & 51,82 & 19,80 & 39,15 & 28,32 & 41,67 \\
\hline CVe\% & 32,69 & 54,45 & 30,18 & 52,86 & 46,11 & 61,04 \\
\hline $\mathrm{CVr}$ & 0,83 & 0,95 & 0,66 & 0,74 & 0,61 & 0,68 \\
\hline Média & 43,29 & 64,82 & 35,63 & 58,39 & 50,27 & 74,37 \\
\hline
\end{tabular}

Sendo os parâmetros: $\mathbf{h}^{\mathbf{2}} \mathbf{g}$ : herdabilidade de parcelas individuais no sentido amplo, ou seja, dos efeitos genotípicos totais; $\mathbf{c}^{\mathbf{2}} \mathbf{b l o c}$ : coeficiente de determinação dos efeitos de bloco; $\mathbf{h}^{2} \mathbf{m c}$ : herdabilidade ajustada da média de genótipo; Acclon: acurácia da seleção de genótipos; CVgi\%: coeficiente de variação genotípica individual; CVe\%: coeficiente de variação residual; CVr: coeficiente de variação relativa; Média geral do experimento.

Para todas as características o teste LRT apontou significância estatística para a herdabilidade (tabela de Deviance em materiais suplementares). 
Considerar este fator é importante, pois permite detectar qual o local, dentre os avaliados, é o mais adequado para se realizar a seleção de indivíduos em um programa de melhoramento.

Os valores de herdabilidade de parcelas individuais no sentido amplo $\left(\mathrm{h}^{2} \mathrm{~g}\right)$ variaram entre 0,26 e 0,47 e são considerados moderados (RESENDE, 2002). Este resultado pode ser justificado pelo fato de que a análise individual utiliza os dados de cada idade e local separadamente, e por ela considerar as variâncias obtidas para cada ambiente durante o cálculo da herdabilidade (MALOSETTI et al., 2013). Outro resultado que corroborou com os valores obtidos para a herdabilidade foi o do coeficiente de variação relativa $(\mathrm{CVr})$. As relações demonstradas por este coeficiente apresentaram valores próximos a 1 (variaram entre 0,61 e 0,95). Estes valores indicam que houve menor influência dos resíduos quando cada clone foi avaliado em seus hortos e também, que esta análise resulta em maior efeito genético na expressão dos caracteres.

O sítio que apresentou maiores valores de CVr foi o Capivara $(0,83$ e 0,95, aos 3 e 9 anos, respectivamente), o que indica haver maior variabilidade genética entre os indivíduos nesta área. É importante considerar, que enquanto Capivara possui maior variabilidade, São João possui maiores médias de produtividade, devendo estes dois resultados serem ponderados durante a tomada de decisão para a seleção dos melhores clones nestes locais. Os valores genéticos para os locais em cada idade ultrapassaram 94\% (Tabela 3), portanto, foram preditos com alta acurácia (RESENDE; DUARTE, 2007). Sendo a acurácia o parâmetro mais adequado para demonstrar a qualidade experimental e eficiência do melhoramento, os valores encontrados ratificam que o delineamento empregado e o número de repetições (30) nos testes clonais foram adequados para garantir alta confiabilidade dos resultados.

\section{Parâmetros genéticos da análise conjunta de interação $\mathbf{G} \times \mathbf{A}$ em diferentes idades}

Constatou-se baixos valores de herdabilidade de parcelas individuais no sentido amplo $(0,01$ a 0,12$)$, devido à diferença entre os locais e de idades, que acarretaram em distribuição da variância (Tabela 4) devido à complexidade do modelo estatístico. Esta complexidade pode ser causada pelo maior número de fatores na análise, como as diferentes condições edafoclimáticas e idades. Sendo assim, foi importante a realização prévia das análises individuais, para facilitar a visualização da variabilidade entre os locais e idades, antes de se avaliar os parâmetros genéticos em análise conjunta.

A avaliação da correlação genotípica entre locais é importante para o entendimento do desenvolvimento dos povoamentos nos diferentes sítios da rede experimental. As correlações para a variável IMA, através dos locais $(\mathrm{Rgl}=0,49)$ e através dos locais e medições $($ Rglocm $=0,29)$ foram baixas. Dessa maneira, é possível concluir que a seleção de clones superiores nos locais avaliados

Tabela 4. Estimativas de parâmetros genéticos da análise conjunta entre os ambientes e entre as idades de 3 e 9 anos,

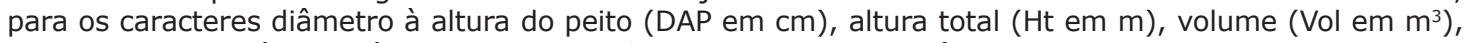
incremento volumétrico médio anual (IMA, $\mathrm{m}^{3}$. ha-1. ano $^{-1}$ ) e sobrevivência de clones de eucalipto avaliados.

Table 4. Estimates of genetic parameters of the joint analysis between environments at ages of 3 and 9 years, for traits diameter at breast height (DAP in $\mathrm{cm}$ ), total height $(\mathrm{Ht}$ in $\mathrm{m})$, volume (Vol in $\left.\mathrm{m}^{3}\right)$, mean annual increment (MAI in $\mathrm{m}^{3} \cdot \mathrm{ha}^{-1} \cdot \mathrm{yr}^{-1}$ ) and survival of Eucalyptus clones evaluated.

\begin{tabular}{cccccc}
\hline Parâmetros & DAP & Ht & Vol & IMA & Sobrevivência \\
\hline$h^{2} g$ & 0,12 & 0,06 & 0,04 & 0,11 & 0,01 \\
$h^{2} m g$ & 0,58 & 0,44 & 0,26 & 0,53 & 0,40 \\
Acgen & 0,76 & 0,66 & 0,51 & 0,73 & 0,63 \\
$c^{2} g l$ & 0,10 & 0,08 & 0,04 & 0,11 & 0,04 \\
$c^{2}$ glm & 0,05 & 0,05 & 0,13 & 0,06 & 0,00 \\
$c^{2}$ perm & 0,39 & 0,33 & 0,15 & 0,36 & 0,72 \\
Rgl & 0,54 & 0,42 & 0,49 & 0,49 & 0,21 \\
Rglocm & 0,33 & 0,22 & 0,11 & 0,29 & 0,20 \\
Média & 16,72 & 20,57 & 0,28 & 55,99 & 0,87 \\
\hline
\end{tabular}

Sendo os parâmetros: $\mathbf{h}^{\mathbf{2}} \mathbf{g}$ : herdabilidade de parcelas individuais no sentido amplo, ou seja, dos efeitos genotípicos totais; $\mathbf{h} \mathbf{2 m g : ~ h e r d a b i l i d a d e ~}$ no sentido amplo da média de genótipos; Acgen: acurácia da seleção de genótipos; $\mathbf{c}^{2} \mathbf{g l}$ : coeficiente de determinação dos efeitos da interação genótipo x locais; $\mathbf{c}^{2} \mathbf{g m}$ : coeficiente de determinação dos efeitos da interação genótipos $\mathrm{x}$ medições; $\mathbf{c}^{2} \mathbf{g l m}$ : coeficiente de determinação dos efeitos da interação genótipo x locais x medições; $\mathbf{c}^{2}$ perm: coeficiente de determinação dos efeitos permanentes de parcela; $\mathbf{R}$ : repetibilidade individual; Rgl: correlação genotípica através dos locais, válida para qualquer medição; Rgm: correlação genotípica através das medições, válida para qualquer local; Rglocm: correlação genotípica através dos locais e medições; Média geral do experimento.

Para todas as características o teste LRT apontou significância estatística para a herdabilidade (tabela de Deviance em materiais suplementares). 
não pode ocorrer de forma generalizada, tanto ao nível de locais como de locais e anos de medição. A acurácia de predição para estas estimativas relacionadas ao IMA foi igual a 0,73, sendo este valor considerado alto segundo Resende e Duarte (2007).

\section{Zonas de melhoramento}

As correlações genéticas entre os ambientes para o caráter IMA, aos 3 e 9 anos, respectivamente, foram: Capivara e Fortaleza (0,48 e 0,62); Capivara e São João (0,53 e 0,57); Fortaleza e São João $(0,62$ e 0,72$)$. A partir dos valores obtidos é possível constatar que aos 3 anos as correlações são baixas (RESENDE, 2005), demonstrando que existem influências diferenciadas dos locais para o desempenho dos genótipos. Além disso, é possível constatar que a correlação aumenta para todas as relações entre ambientes na idade de 9 anos.

Ainda nesta idade, apenas a correlação entre São João $\times$ Fortaleza atingiu valor superior a 0,70 (correlação $=0,72)$, que é o valor de referência para indicar que estes locais formam uma única zona de melhoramento, de acordo com Resende e Duarte (2007). Aos 9 anos, as correlações entre Capivara $\times$ São João e Capivara $\times$ Fortaleza apresentaram valores considerados baixos. Dessa forma, conclui-se que aos 9 anos há apenas duas zonas de melhoramento entre os ambientes, sendo estas São João-Fortaleza e Capivara, enquanto que aos 3 anos podem ser constatadas três zonas, que são representadas por cada sítio. O resultado encontrado demonstra, que apesar da proximidade entre os ambientes Fortaleza e Capivara, demonstrada pela Figura 1, os fatores edafoclimáticos podem ser relevantes para a criação de zonas de melhoramento.

Levando em consideração estes aspectos, as zonas de melhoramento encontradas neste trabalho, corroboram com os resultados obtidos anteriormente por Nunes et al. (2016). Esses autores demonstraram que o sítio Capivara apresenta características de solo diferente dos demais e estabeleceu as mesmas zonas de melhoramento encontradas na presente pesquisa. Esta diferenciação pode ter ocorrido devido à textura do solo deste local ser do tipo arenosa/argilosa (Tabela 1), o que possivelmente influenciou no desenvolvimento diferenciado dos genótipos neste ambiente em relação aos demais locais.

As zonas de melhoramento são comumente criadas para subdividir a área total do experimento e minimizar os efeitos da interação $\mathrm{G} \times \mathrm{A}$ dentro dessas zonas (ELDRIDGE et al., 1994; RESENDE, 2005; SOUZA et al., 1992). Cada zona de melhoramento é caracterizada por não possuir interação $\mathrm{G} \times$ A complexa, ou seja, demanda apenas um programa de melhoramento específico, que possibilita a obtenção de ganhos genéticos maximizados em cada área (MARTINEZ et al., 2012; NUNES et al., 2016; RESENDE, 2005).

Avaliar a formação de zonas de melhoramento é importante durante o processo de recomendação de novos clones para plantio comercial, pois havendo diferentes níveis de correlações genotípicas entre os ambientes, devem ser realizados diferentes tipos de indicações para plantio em cada área, de acordo com a correlação apresentada. Com os resultados obtidos, em relação aos parâmetros genéticos e à formação de zonas de melhoramento, é possível concluir que a interação entre os genótipos e os ambientes se modifica com o passar dos anos para o caráter IMA. Pode-se afirmar, portanto, que com o passar do tempo, a influência do ambiente sobre o desempenho dos clones diminuiu, impactando na forma como os clones devem ser selecionados.

\section{Ganho genético}

A avaliação dos ganhos genéticos foi realizada para a seleção dos melhores genótipos, a partir da comparação entre seus valores genotípicos preditos livres de interação $(\mathrm{u}+\mathrm{g})$ e dos valores genotípicos preditos acrescidos da interação média entre ambientes $(\mathrm{u}+\mathrm{g}+\mathrm{gem})$. O primeiro índice $(\mathrm{u}+\mathrm{g})$, é utilizado para a recomendação de genótipos para diferentes áreas da rede experimental. O segundo (u+g+gem), considera uma interação média entre os ambientes estudados, sendo, portanto, utilizado para recomendação dos clones na rede experimental de forma generalizada.

O ordenamento dos 10 melhores clones na seleção conjunta para o IMA, entre os 3 ambientes (Cambará, São João e Fortaleza) e as idades de 3 e 9 anos é visto na Tabela 5. Os cinco melhores clones apresentaram desempenho superior à testemunha, com ganho genético acumulado para $\mathrm{u}+\mathrm{g}$ igual a $21 \%$ e para $\mathrm{u}+\mathrm{g}+\mathrm{gem}$ igual a $27 \%$. Esta superioridade em relação ao clone testemunha (32864) demonstrou a potencialidade de se obter materiais superiores a partir de novos testes clonais. Ao se comparar o desempenho destes mesmos clones com a média geral, obtida a partir 
Castro et al. - Se Comportamento da interação genótipos por locais aos três e

nove anos em clones de eucalipto

Tabela 5. Ranqueamento e ganhos genéticos dos clones, para o caráter incremento volumétrico médio anual (IMA, $\left.\mathrm{m}^{3} \cdot \mathrm{ha}^{-1} \cdot \mathrm{ano}^{-1}\right)$, em clones de eucalipto após a análise conjunta entre todos os ambientes e as idades.

Table 5. Genetic rankings and genetic gains of the clones, using the trait mean annual increment $\left(M A I, m^{3} \cdot h^{-1} \cdot y r^{-1}\right)$, addressing in Eucalyptus clones after a joint analysis between all the environments and the ages.

\begin{tabular}{|c|c|c|c|c|c|c|c|}
\hline \multicolumn{8}{|c|}{ IMA } \\
\hline \multirow[b]{2}{*}{ Ordem } & \multirow[b]{2}{*}{ Genótipos } & \multirow[b]{2}{*}{$u+g$} & \multirow[b]{2}{*}{$\mathrm{u}+\mathrm{g}+\mathrm{gem}$} & \multicolumn{2}{|c|}{$u+g$} & \multicolumn{2}{|c|}{$u+g+g e m$} \\
\hline & & & & $\begin{array}{l}\text { Ganho em } \\
\text { relação à média } \\
\text { da população }\end{array}$ & $\begin{array}{l}\text { Ganho em } \\
\text { relação à } \\
\text { testemunha }\end{array}$ & $\begin{array}{l}\text { Ganho em } \\
\text { relação à média } \\
\text { da população }\end{array}$ & $\begin{array}{l}\text { Ganho em } \\
\text { relação à } \\
\text { testemunha }\end{array}$ \\
\hline 1 & 39416 & 82,67 & 91,95 & $47,65 \%$ & $31,07 \%$ & $64,23 \%$ & $40,31 \%$ \\
\hline 2 & 39096 & 75,67 & 82,52 & $35,16 \%$ & $19,99 \%$ & $47,39 \%$ & $25,93 \%$ \\
\hline 3 & 39002 & 74,81 & 81,36 & $33,62 \%$ & $18,61 \%$ & $45,31 \%$ & $24,15 \%$ \\
\hline 4 & 39032 & 74,09 & 80,39 & $32,34 \%$ & $17,48 \%$ & $43,59 \%$ & $22,67 \%$ \\
\hline 5 & 39018 & 73,69 & 79,84 & $31,61 \%$ & $16,83 \%$ & $42,61 \%$ & $21,84 \%$ \\
\hline 6 & 39654 & 73,67 & 79,82 & $31,59 \%$ & $16,81 \%$ & $42,57 \%$ & $21,81 \%$ \\
\hline 7 & 38935 & 73,01 & 78,93 & $30,40 \%$ & $15,76 \%$ & $40,98 \%$ & $20,44 \%$ \\
\hline 8 & 39700 & 72,32 & 78,00 & $29,17 \%$ & $14,66 \%$ & $39,31 \%$ & $19,02 \%$ \\
\hline 9 & 39423 & 72,14 & 77,76 & $28,85 \%$ & $14,38 \%$ & $38,89 \%$ & $18,66 \%$ \\
\hline 10 & 39251 & 71,42 & 76,78 & $27,56 \%$ & $13,23 \%$ & $37,14 \%$ & $17,17 \%$ \\
\hline 62 & 32864 & 63.07 & 6553 & $1265 \%$ & $0.00 \%$ & $17.05 \%$ & $0.00 \%$ \\
\hline
\end{tabular}

Ganho acumulado dos 5 melhores genótipos para u+g em relação à testemunha: $21 \%$

Ganho acumulado dos 5 melhores genótipos para $u+g+g e m$ em relação à testemunha: $27 \%$

da média de ganho de todos os clones, obteve-se maiores porcentagens de ganhos do que os que foram obtidos anteriormente, quando estes foram relacionados com a testemunha. Neste sentido, os ganhos genéticos acumulados com a seleção dos melhores genótipos, comparados à média geral, foram iguais a $36 \%$ e $49 \%$ para $\mathrm{u}+\mathrm{g}$ e $\mathrm{u}+\mathrm{g}+\mathrm{gem}$, respectivamente.

A obtenção de ganhos genéticos em programas de melhoramento de eucalipto tem se tornado cada vez mais difícil, uma vez que genótipos altamente adaptados são gerados a cada ano. O uso de procedimentos analíticos mais refinados, como o emprego de modelos lineares mistos, para o estudo mais detalhado dos componentes da média e da variância dos caracteres, tem possibilitado a identificação de genótipos superiores às testemunhas comerciais (MAIA et al., 2009).

\section{Adaptabilidade e estabilidade}

Os 10 melhores clones para adaptabilidade, estabilidade e produtividade, simultaneamente, encontram-se na Tabela 6 . O desempenho da testemunha foi comparado ao dos demais clones do ranqueamento. Os cinco melhores clones apresentaram ganho acumulado de $22 \%$ sobre a testemunha e de $43 \%$ em relação à média geral dos experimentos, com base na MHPRVG*MG.

A avaliação da interação $\mathrm{G} \times$ A não é suficiente para a correta interpretação do comportamento dos genótipos em diferentes condições ambientais (CRUZ; REGAZZI; CARNEIRO, 2012). Sendo assim, análises de adaptabilidade e estabilidade são de grande importância no melhoramento florestal, pois podem ser utilizadas como complemento ao estudo relacionado à estratificação ambiental (PINTO JÚNIOR et al., 2011; SANTOS et al., 2016, 2013). Neste sentido, é importante avaliar os genótipos que possuem constância em seu desempenho e que respondem de maneira favorável às variações ambientais, em condições amplas ou específicas (CRUZ; REGAZZI; CARNEIRO, 2012; NUNES et al., 2016).

Ao se avaliar a viabilidade de seleção dos cinco melhores clones com base no desempenho médio que estes tiveram nos três ambientes, em relação aos valores genotípicos livres da interação, observa-se que o clone 39416 apresentou o melhor desempenho no ordenamento da MHPRVG*MG. Este clone esteve no primeiro lugar dos ranqueamentos relacionados aos três quesitos avaliados, além de ter sido o clone a obter maior ganho genético (Tabela 5). O 39416 demonstrou ser o principal material genético com potencial para recomendação. Outros dois clones (39032 e 39096) também apresentaram boas classificações (Tabelas 5 e 6), pois ambos se mantiveram entre as quatro primeiras colocações. 
Tabela 6. Ordenamento da adaptabilidade de valores genéticos (PRVG), estabilidade de valores genéticos (MHVG) e estabilidade e adaptabilidade simultaneamente (MHPRVG) para o caráter incremento volumétrico médio anual (IMA, $\mathrm{m}^{3}$.ha-1. ano ${ }^{-1}$ ) dos clones de eucalipto, após análise conjunta entre todos os ambientes e idades.

Table 6. Ranking of adaptability of genetic values (PRVG), stability of genetic values (MHVG) and stability and adaptability simultaneously (MHPRVG) for the mean annual increment (MAI, $\mathrm{m}^{3} \cdot \mathrm{ha}^{-1} \cdot \mathrm{yr}^{-1}$ ) of the Eucalyptus clones after a joint analysis between all environments and ages.

\begin{tabular}{|c|c|c|c|c|c|c|c|c|c|c|}
\hline \multicolumn{11}{|c|}{ IMA } \\
\hline \multicolumn{4}{|c|}{ Adaptabilidade } & \multicolumn{3}{|c|}{ Estabilidade } & \multicolumn{4}{|c|}{ Adaptabilidade e Estabilidade } \\
\hline Ordem & Genótipos & PRVG & PRVG*MG & Ordem & Genótipo & MHVG & Ordem & Genótipo & MHPRVG & MHPRVG*MG \\
\hline 1 & 39416 & 1,65 & 92,19 & 1 & 39416 & 90,84 & 1 & 39416 & 1,64 & 91,99 \\
\hline 2 & 39002 & 1,48 & 82,71 & 2 & 39654 & 79,33 & 2 & 39032 & 1,43 & 80,14 \\
\hline 3 & 39654 & 1,46 & 81,48 & 3 & 39032 & 79,10 & 3 & 39096 & 1,42 & 79,28 \\
\hline 4 & 39096 & 1,45 & 81,38 & 4 & 39002 & 78,97 & 4 & 38935 & 1,41 & 79,01 \\
\hline 5 & 39032 & 1,44 & 80,49 & 5 & 38935 & 77,94 & 5 & 39654 & 1,41 & 78,79 \\
\hline 6 & 39018 & 1,41 & 79,17 & 6 & 39018 & 76,94 & 6 & 39018 & 1,41 & 78,68 \\
\hline 7 & 38935 & 1,41 & 79,02 & 7 & 39096 & 76,81 & 7 & 39002 & 1,40 & 78,65 \\
\hline 8 & 39700 & 1,39 & 77,69 & 8 & 39700 & 75,47 & 8 & 39700 & 1,38 & 77,01 \\
\hline 9 & 39423 & 1,38 & 77,39 & 9 & 39423 & 74,96 & 9 & 39423 & 1,37 & 76,57 \\
\hline 10 & 39259 & 1,37 & 76,69 & 10 & 39095 & 73,92 & 10 & 39251 & 1,35 & 75,58 \\
\hline \multicolumn{4}{|c|}{$\cdots$} & & $\ldots$ & & & & $\ldots$ & \\
\hline 61 & 32864 & 1,16 & 65,06 & 60 & 32864 & 62,98 & 58 & 32864 & 1,15 & 64,47 \\
\hline
\end{tabular}

\section{CONCLUSÃO}

O padrão de interação $\mathrm{G} \times \mathrm{A}$ se modificou dos 3 para os 9 anos, devido à uniformização do desempenho dos clones e à melhor adaptação destes às variações ambientais, em idades mais avançadas. Aos 3 anos foram estabelecidas três zonas de melhoramento, representadas por cada sítio, enquanto que aos 9 formaram-se duas zonas, sendo estas São João-Fortaleza e Capivara.

\section{AGRADECIMENTOS}

Os autores agradecem à FAPEMIG, CAPES e CNPq pela concessão de bolsas e à empresa CMPC Celulose Riograndense por conceder os dados.

\section{REFERÊNCIAS BIBLIOGRÁFICAS}

ARAÚJO, M. J.; DIAS, D. C.; SCARPINATI, E. A.; PAULA, R. C. Número de repetições, de plantas por parcela e de avaliações para testes clonais de eucalipto. Pesquisa Agropecuária Brasileira, Brasília, v. 50, n. 10, p. 923-931, 2015.

ASSIS, T. F.; ABAD, J. I. M.; AGUIAR, A. M. Melhoramento genético do eucalipto. Informe Agropecuário, Belo Horizonte, v. 12, n. 141, p. 225-247, 1996.

BIRK, E. M.; TURNER, J. Response of flooded gum (E. grandis) to intensive cultural treatments: biomass and nutrient content of eucalypt plantations and native forests. Forest Ecology and Management, Amsterdam, v. 47, n. $1-4$, p. $1-28,1992$.

CASTRO, C. A. O.; RESENDE, R. T.; BHERING, L. L.; CRUZ, C. D. Brief history of Eucalyptus breeding in Brazil under perspective of biometric advances. Ciência Rural, Santa Maria, v. 46, n. 9, p. 1585-1593, 2016.

CRUZ, C. D.; REGAZZI, A. J.; CARNEIRO, P. C. S. Modelos biométricos aplicados ao melhoramento genético. Viçosa: Editora UFV, 2012. 514 p.

ELDRIDGE, K; DAVIDSON, J.; HARWOOD, C.;WYK, G.V. Eucalypt domestication and breeding. New York: Oxford University Press, 1994, 288 p.

FALCONER, D. S.; MACKAY, T.; FRANKHAM, R. Introduction to quantitative genetics. Trends in Genetics, v. 12, n. 7, p. 280, 1996. 
Castro et al. - Se Comportamento da interação genótipos por locais aos três e

nove anos em clones de eucalipto

FONSECA, S. M. F.; RESENDE, M. D. V.; ALFENAS, A. C; GUIMARÃES, L. M. S.; ASSIS, T. F.; GRATTAPAGLIA, D. Manual prático de melhoramento genético de eucalipto. Viçosa: Editora UFV, 2010. 200 p.

GARbugliO, D. D.; GerAGE, A. C.; ARAÚJO, P. M.; JUNIOR, N. D. S. F.; SHIOGA, P. S. Análise de fatores e regressão bissegmentada em estudos de estratificação ambiental e adaptabilidade em milho. Pesquisa Agropecuária Brasileira, Brasília, v. 42, n. 2, p. 183-191, 2007.

HARDNER, C.; DIETERS, M.; DELACY, I.; NEAL, J.; FLETCHER, S.; DALE, G.; BASFORD, K. Identifying deployment zones for Eucalyptus camaldulensis $\mathrm{x}$ E. globulus and $\mathrm{x}$ E. grandis hybrids using factor analytic modelling of genotype by environment interaction. Australian Forestry, Melbourne, v. 74, n. 1, p. 30-35, 2011.

MAIA, M. C. C.; RESENDE, M. D. V.; PAIVA, J. R.; CAVALCANTI, J. J. V.; BARROS, L. M. B. Seleção simultânea para produção, adaptabilidade e estabilidade genotípicas em clones de cajueiro, via modelos mistos. Pesquisa Agropecuária Tropical, Goiânia, v. 39, n. 1, p. 43-50, 2009.

MALOSETTI, M.; RIBAUT, J. M.; VAN EEUWIJK, F. A. The statistical analysis of multi-environment data: modeling genotype-by-environment interaction and its genetic basis. Frontiers in physiology, v. 4, p. 44, 2013.

MARTINEZ, D. T.; RESENDE, M. D. V.; COSTA, R. B.; HIGA, A. R.; SANTOS, G. A.; FIER, I. S. N. Estudo da interação genótipo $\mathrm{x}$ ambiente em progênies de Pinus taeda por meio da análise de parâmetros genéticos. Floresta, Curitiba, v. 42, n. 3, p. 539-552, 2012.

NUNES, A. C. P.; SANTOS, G. A.; RESENDE, M. D. V.; SILVA, L. D.; HIGA, A.; ASSIS, T. F. Estabelecimento de zonas de melhoramento para clones de eucalipto no Rio Grande do Sul. Scientia Forestalis, Piracicaba, v. 44, n. 111, p. 563-574, 2016.

NUNES, G. H. S.; REZENDE, G. D. S. P.; RAMALHO, M. A. P.; SANTOS, J. B. Implicações da interação genótipos x ambientes na seleção de clones de eucalipto. Cerne, Lavras, v. 8, n. 1, p. 49-58, 2002.

ODA, S.; MENCK, A. L. M.; VENCOVSKY, R. Problemas no melhoramento genético clássico do eucalipto em função da alta intensidade de seleção. IPEF, Piracicaba, v. 41, n. 42, p. 8-17, 1989.

PINTO JÚNIOR, J. E.; STURION, J. A.; RESENDE, M. D. V.; JÚNIOR, P. R. Avaliação Simultânea de Produtividade, Adaptabilidade e Estabilidade Genotípica de Eucalyptus grandis em Distintos Ambientes do Estado de São Paulo. Pesquisa Florestal Brasileira, Colombo, n. 53, p. 79-108, 2011.

PIRES, I. E.; RESENDE, M. D. V.; SILVA, R. L.; JÚNIOR, M. F. R. R. Genética florestal. Viçosa: Arka, 2011.318 p.

RESENDE, M. D. V. Software Selegen-REML/BLUP: a useful tool for plant breeding. Crop Breeding and Applied Biotechnology. v. 16, p. 330-339, 2016.

RESENDE, M. D. V. Melhoramento de Essências Florestais. In: BOREM, A. Melhoramento de Espécies Cultivadas. Viçosa: Editora UFV, 2005. p. 717-780.

RESENDE, M. D. V. Novas abordagens estatísticas na análise de experimentos e campo. Colombo: Embrapa Florestas, 2004. 60 p. (Documentos, 100).

RESENDE, M. D. V. Genética biométrica e estatística no melhoramento de plantas perenes. Colombo: Embrapa Florestas, 2002. 975 p.

RESENDE, M. D. V.; DUARTE, J. B. Precisão e controle de qualidade em experimentos de avaliação de cultivares. Pesquisa Agropecuária Tropical, Goiânia,v. 37, n. 3, p. 182-194, 2007.

RESENDE, R. T.; RESENDE, M. D. V.; SILVA, F. F.; TAKAHASHI, E. K. Acurácia preditiva de testes clonais de Eucalyptus spp. utilizando efeitos aditivos do parentesco e validação cruzada. Scientia Forestalis, Piracicaba, v. 45, n. 113, p. 39-47, 2017.

ROSADO, A. M.; ROSADO, T. B.; ALVES, A. A.; LAVIOLA, B. G.; BHERING, L. L. Seleção simultânea de clones de eucalipto de acordo com produtividade, estabilidade e adaptabilidade. Pesquisa Agropecuária Brasileira, Brasília, v. 47, n. 7, p. 964-971, 2012.

SANTOS, G. A.; NUNES, A. C. P.; RESENDE, M. D. V.; SILVA, L. D.; HIGA, A.; ASSIS, T. F. An index combining volume and Pilodyn penetration to study stability and adaptability of Eucalyptus multi-species hybrids in Rio Grande do Sul, Brazil. Australian Forestry, Melbourne, v. 79, n. 4, p. 248-255, 2016. 
SANTOS, G. A.; RESENDE, M. D. V.; SILVA, L. D.; HIGA, A.; ASSIS, T. Adaptabilidade de híbridos multiespécies de Eucalyptus ao Estado do Rio Grande do Sul. Revista Árvore, Viçosa, v. 37, n. 4, p. 759-769, 2013.

SCHUMACHER, F. X.; HALL, F. S. Logarithmic expression of timber-tree volume. Journal of Agricultural Research, Washington, v.47, n.9, p.719-734, 1933.

SOUZA, S. M.; RESENDE, M. D. V.; SILVA, H. D.; HIGA, A. R. Variabilidade genética e interação genótipo x ambiente envolvendo procedências de Eucalyptus cloeziana F. Muell., em diferentes regiões do Brasil. Revista Arvore, Viçosa, v. 16, n. 1, p. 1-17, 1992.

STURION, J. A.; RESENDE, M. D. V. Seleção de progênies de erva-mate (Ilex paraguariensis St. Hill.) para a produtividade, estabilidade e adaptabilidade temporal de massa foliar. Pesquisa Florestal Brasileira, Colombo, n. 50, p. 37-51, 2005.

VENCOVSKY, R.; BARRIGA, P. Genética biométrica no fitomelhoramento. Ribeirão Preto: Sociedade Brasileira de Genética, 1992. 416 p.

Recebido em:18/09/2017

Aceito em: 03/05/2018 